\title{
Importancia del estudio del cuerpo en los contextos de la salud-enfermedad y responsabilidad ética del investigador social
}

\author{
Importance of studying the body in health-disease contexts \\ and the ethical responsibility of social researchers
}

Maritza Charry Higuera*

\section{Resumen}

Este texto intenta plantear algunas de las tendencias teóricas sobre la comprensión del cuerpo como categoría analítica y las implicaciones éticas del investigador social en los contextos de salud-enfermedad. En primera instancia, se presentarán estas tendencias en el abordaje del cuerpo como objeto de conocimiento, y en segunda instancia se introducirán las principales implicaciones de carácter ético y la responsabilidad social del investigador frente a la realidad de las personas. Para ello es necesario comprender los límites visibles e invisibles entre investigar e intervenir, entendiendo que la palabra es acción y, por tanto, el efecto del testimonio en la vida de las personas es incierto, aun cuando el investigador crea que pueda tener un efecto terapéutico.

Palabras clave: cuerpo, intervención, investigación, ética, testimonio.

\section{Abstract}

This paper seeks to discuss some of the theoretical trends on the body as an analytical category, as well as the social researcher's ethical implications in health-disease contexts. These trends will be initially presented by approaching the body as an object of knowledge, and later the main ethical implications and the social researcher's responsibility before people's

${ }^{*}$ Trabajadora Social, Especialista en Intervención con Familias. Magíster en Intervención Social Universidad del Valle. Docente contratista en la Escuela de Trabajo Social y Desarrollo Humano de la misma Universidad, y Trabajadora Social de Coomeva, Medicina Prepagada. Correo electrónico: maricharry@hotmail.com.

Artículo tipo 1: de investigación científica.

Recibido: 29 de marzo de 2012 Aprobado: 10 de mayo de 2012 
reality will be presented. It is then necessary to understand the visible and invisible boundaries between researching and intervening, considering that language means action, and therefore the effect of testimony on people's lives is uncertain, even if the researcher believes it might have a therapeutic effect.

Keywords: body, intervention, research, ethics, testimony.

Sumario: 1. Presentación, 2. Comprensión del cuerpo en contextos salud-enfermedad, 3. Implicaciones éticas del investigador en los procesos de investigación social, 4. Conclusiones y 5. Referencias bibliográficas.

\section{Presentación}

El presente texto es un producto de la reflexión metodológica realizada en la investigación titulada "El cuerpo, entre la salud y la enfermedad: Significados del cuerpo en personas con diabetes mellitus en la ciudad de Cali", realizada durante los años 2010-2011, para optar al título de Magíster en Intervención Social - Énfasis en Familia, en la Facultad de Humanidades, Escuela de Trabajo Social y Desarrollo Humano de la Universidad del Valle.

La investigación surgió de las experiencias de intervención familiar en el área de la salud con personas que padecían diabetes mellitus y buscó trascender del objeto de intervención a la profundización del conocimiento del cuerpo en contextos de salud-enfermedad. Se partió de perspectivas fenomenológicas y constructivistas, ambas complementarias para el acercamiento comprensivo del cuerpo. Por una parte, en la dimensión constitutiva del cuerpo se materializan las enfermedades, pero, al mismo tiempo, el carácter subjetivo e interpretativo que se le atribuye al cuerpo, a partir de las relaciones con los otros significativos y con el discurso médico predominante, le otorga un énfasis más simbólico en la experimentación corporal ante la enfermedad crónica. En el mismo sentido, ambas perspectivas teóricas permiten comprender el cuerpo desde 
la convergencia de polos que hasta ahora han venido tratándose de manera aislada: naturaleza/cultura, salud/enfermedad, cuerpo/persona.

A continuación se realiza un recorrido teórico del cuerpo como categoría analítica y posteriormente se abordará la reflexión sobre las implicaciones éticas del investigador en los procesos de investigación social.

\section{Comprensión del cuerpo en contextos salud-enfermedad}

Las fuertes consideraciones teóricas y metodológicas del cuerpo como objeto de conocimiento han llevado a plantearlo como una categoría existente antes de la cultura, cuyo esencialismo primario, basado en argumentos biológicos, lleva a una visión materialista del cuerpo, "una suerte de recipiente material, [...] marco biológico necesario para que nuestras actividades sociales sean definidas en el contexto de la realidad" (Salinas, 1994: 86). De otro lado, en las consideraciones sociales y culturales del cuerpo, la dimensión biológica pasa a un segundo plano, porque la acción de la cultura y de lo social interactúa y reconstruye al individuo constantemente, modificando y recreando "esas realidades biológicas" (García, 1994: 45). A continuación se presentan de manera más detallada algunas tendencias teóricas sobre la comprensión del cuerpo.

Los desarrollos de la ciencia moderna y los aportes filosóficos de Descartes fundaron una visión dicotómica sobre el cuerpo y la mente: "sustancia pensante -res cogitans- y sustancia física -res extensa-" (Reyes y Eroza, 2008), que arraigó el énfasis material del cuerpo y que fue consolidando las diversas teorías desarrolladas sobre el ser humano, dividiendo el estudio de lo mental, lo emocional, el pensamiento y lo social, separadamente de la corporalidad.

Más adelante, los desarrollos del carácter fenomenológico del cuerpo, representado por los trabajos del filósofo francés Maurice MerleauPonty (1984), rompieron con las dicotomías establecidas y heredadas del dualismo cartesiano, considerando el cuerpo en su carácter constitutivo de la existencia y experimentación del mundo. El cuerpo es el que nos permite percibir la realidad y acceder al mundo a través de las sensaciones que producen los sentidos de nuestra constitución corporal; por lo tanto, 
no es posible separar el cuerpo de la existencia humana y de lo que experimentamos del mundo, porque el cuerpo se constituye en el vehículo primordial de comunicación humana con los otros en el mundo (MerleauPonty, 1984). Estas visiones fenomenológicas de la corporalidad se han ido desarrollando en líneas de investigación de sociólogos y antropólogos que se dedican a estudiar el cuerpo desde esta perspectiva; entre ellos están los trabajos contemporáneos de David Le Bretón (2007), sociólogo y antropólogo francés cuya producción literaria sobre el cuerpo retoma los principales aportes de Merleau-Ponty, conformando la antropología del cuerpo y los aspectos culturales en diversos contextos mediáticos de enfermedad y sufrimiento humano.

Asimismo, se destacan los trabajos de las antropólogas Veena Das (1996) en antropología médica e investigaciones en contextos de violencia sobre la experimentación corporal del sufrimiento de mujeres, y de la argentina Silvia Citro (2009), quien se ha dedicado a investigar acerca de la corporalidad como constitutiva de la experiencia vivida.

Arthur Kleinman, psiquiatra y antropólogo de la Universidad de Harvard, es otro de los que retoma el tema del cuerpo y la vivencia de la enfermedad desde una visión fenomenológica (Kleinman, 1988). Para él, el cuerpo es un receptáculo de comunicación simbólica en el que los diversos aspectos de la condición humana son representados a través de las experiencias de sufrimiento y enfermedad. Ante la enfermedad, la concepción del cuerpo cambia, no sólo por el cambio de las sensaciones corporales y subjetivas del ser humano, sino por las construcciones sociales que se hacen del cuerpo y de la enfermedad de acuerdo a la cultura predominante de las sociedades y al momento histórico. En las culturas occidentales, el modelo biomédico existente ayuda a construir una visión cerrada y dicotómica del cuerpo, separándolo de los pensamientos, de las emociones y del mundo; a diferencia de las sociedades no occidentales, en las cuales el cuerpo se percibe en constante interrelación con las emociones, el pensamiento y lo cultural (Kleinman, 1988).

Una tercera tendencia sobre el estudio del cuerpo es la visión foucaultiana, que se ocupa de discutir el peso que las estructuras sociales ejercen sobre el cuerpo. Por ejemplo: los preceptos del cultivo de sí, los mandatos de las 
doctrinas filosóficas platónicas y epicúreas sobre el cuidado del cuerpo y los preceptos y las configuraciones de los discursos sociales, políticos y médicos que el cuerpo recibe. Foucault $(1966,2006)$ cuestiona la visión organística del cuerpo que hace la medicina, configurando el cuerpo como un objeto de observación y de análisis taxonómico-anatómico, que obliga al profesional a abstraerse de la persona y reducirla al plano biológico, enfatizando así más en su carácter material que en el social. El cuerpo es regulado por el logos o saber médico hacia modos establecidos de conducta con uno mismo y con el cuerpo.

En esta misma línea se encuentran los recientes trabajos de Sierra (2009), quien señala que la medicina sigue teniendo un papel domesticador en cuanto al modo de entender y percibir el cuerpo humano. Los avances tecnológicos han ayudado a construir una mirada mecánica del ser humano, en la que el cuerpo se concibe como un almacén de tejidos y órganos que puede ser penetrado bajo el poder de los exámenes “escópicos”, lo que lleva a la construcción de imaginarios de trasparencia y desciframiento humano, instrumentalizando a la persona y llevándose por delante su intimidad, su autonomía y su privacidad. Con la posibilidad de poder observar el cuerpo humano se crean escenarios artificiales en los cuales la mirada del médico puede "viajar" al interior del cuerpo "a través de cada sección material, órgano o tejido del cuerpo humano, desde cualquier perspectiva, porque el cuerpo es en sí mismo un archivo" (Sierra, 2009: 235-236). Por su parte, Suárez (2001) ha considerado que el surgimiento del cuerpo como categoría analítica en la Modernidad ha dirigido su acción hacia la higiene para conservar el cuerpo sano, activo y eficiente; y Pedraza (2003) enfatiza en las cualidades materiales que la medicina ha movilizado, separando el cuerpo de su entorno social y cultural.

Una cuarta tendencia civilizatoria corresponde a los aportes de los sociólogos Norbert Elias (1966) y Marcel Mauss (1979). Elias (1966, 1993) se ocupó de estudiar los procesos de civilización que las sociedades occidentales han desarrollado a partir de la Edad Media. Los seres humanos y su cuerpo se han visto sometidos a una serie de imposiciones y restricciones limitadas que atenúan los excesos corporales y afectan el tipo de relación social que se establece entre los seres humanos. Este 
proceso civilizatorio, consolidado en las sociedades cortesanas durante los siglos XIV y XV, ha logrado modificar las estructuras sociales y mentales de las sociedades hacia la automatización de ciertos actos y comportamientos corporales de los seres humanos, creándose "un muro afectivo" entre el hombre y su propio cuerpo, y luego entre los cuerpos de los hombres, estableciéndose relaciones sociales que se basan más en la distancia que en la cercanía corporal. Comer con cubiertos, hacer las necesidades fisiológicas y tener sexo en espacios privados son ejemplos de la manera como estas imposiciones culturales han permeado las estructuras sociales y mentales, logrando ser parte de las construcciones valorativas de las relaciones sociales. Actualmente es necesario, psicológicamente, mantener la distancia física entre los cuerpos de los hombres, lo que antes era innecesario: se comía de un mismo plato con los dedos, se dormía en la misma cama con los hijos, los cuerpos enfermos y olorosos se juntaban; ahora se han tejido relaciones sociales distantes corporalmente hablando, se percibe el cuerpo y sus funciones de manera desagradable, se toma distancia de los enfermos y se aísla el cuerpo del hombre a tal punto que se objetiviza y se trata como un objeto de consumo (Elias, 1993).

Por su parte, Mauss (1979) realizó diversas investigaciones en las que analizó algunos hechos que las personas consideran como normales o naturales, categorizándolos como técnicas corporales o formas en las que los hombres de cada sociedad hacen uso de su cuerpo en forma acostumbrada. Estas actuaciones pueden ser aprendidas consciente o inconscientemente: la natación, el marchar, el caminar, y una vez adquiridas son difíciles de desprenderse de uno mismo, pues dependen de las costumbres de la sociedad y de su idiosincrasia; las técnicas corporales no son el resultado de mecanismos puramente individuales y físicos, sino que están condicionadas, transmitidas y aprendidas por los procesos educativos, producto de una interrelación compleja entre tres tipos de realidad: el componente físico y mecánico del cuerpo, los aspectos psicológicos y los procesos sociales.

Finalmente, se encuentra la tendencia cotidiana sobre el cuerpo, representada por los desarrollos de Michel de Certeau, quien considera que el cuerpo se construye desde el discurso social y en él se inscriben las 
leyes y las normas de la sociedad a partir de unos individuos que creen en esos discursos y que producen prácticas que legitiman esos discursos: "Hacer creer es hacer hacer" y viceversa; esta capacidad social circular hace maquinar los cuerpos y a su vez creer que lo se hace es válido, encarnándose prácticas físicas sobre el cuerpo, que se creen "reales". Se cree aquello que uno supone que es real. Pero lo real ya está determinado por la ley -la norma social- que ya ha ejercido un efecto sobre el cuerpo y que sin darnos cuenta hace que creamos y practiquemos con nuestro cuerpo la encarnación de aquellas leyes sociales. Encarnación que se da cuando surge el relato que legitima esas prácticas corporales: "los antiguos lo practicaron", o también "otros lo creyeron e hicieron", o "tú mismo llevas en tu cuerpo mi firma", porque el discurso normativo sólo funciona cuando se ha convertido en relato; es decir, cuando otros ya lo han vivido, cuando hay historia situada en un contexto, contada por otros y por sus cuerpos (2000: 160-161). El cuerpo es intervenido por estos discursos que buscan corregirlo, ya sea por el déficit o por el exceso, por la enfermedad o por la estética; según las normas sociales, aun los alimentos que se seleccionan son evaluados y regulados según las tradiciones, buscando moldear los cuerpos al alimentarlos.

Afortunadamente, en la frontera muy extrema de estos discursos, dice De Certeau (2000), quedan posibilidades de escaparse a tales determinaciones invisibles, y surgen cuerpos de niños maleducados, de enfermos o de personas con "falta de modales" que muestran la recia necesidad y las posibilidades que tienen las personas de crear prácticas cotidianas que reconfiguran de manera única y particular estas determinaciones sociales - producto de los sistemas de producción-. Esto implica que aunque los usuarios consumidores de los sistemas de producción estén condenados a la pasividad y a la disciplina, se resisten a sus determinaciones sociales a través de sus prácticas cotidianas o de sus esquemas de acción individuales. Considerando la existencia de una segunda fabricación personal, representada en el nivel de autonomía y de libertad de las personas para hacer uso de esos productos de consumo ya establecidos e impuestos por el orden económico dominante, estos procesos poiéticos, de creación, de "maneras de emplear", manipular o utilizar los productos logran "liberar" al cuerpo de tales discursos sociales. 
De acuerdo a las tendencias teóricas anteriormente expresadas, se hace necesario reconocer la importancia de concebir al cuerpo como un campo de fuerzas cuyas dimensiones físico-natural y socio-cultural están trabadas mutuamente, no son paralelas, sino que se constituyen una a la otra. Como lo plantea García: El cuerpo es "carne y hueso", y al mismo tiempo es una entidad social que "tenemos" y que "somos"; el cuerpo es "individual y único, pero también es común con toda la humanidad". La acción de la cultura y lo social conforma al cuerpo constantemente, modificando y recreando esas "realidades biológicas", sujetas a diversos significados atribuidos a su comportamiento (García, 1994: 45).

Esta visión del cuerpo como constitutivo de lo humano nos permite comprender que tanto lo biológico como lo social están irremediablemente trabados el uno al otro y son inseparables. Como lo afirma Merleau-Ponty, la existencia del cuerpo se constituye en el punto intermedio entre lo psíquico y lo fisiológico:

El hombre concretamente tomado no es un psiquismo conexo a un organismo, sino este vaivén de la existencia ora se deja ser corpórea y ora remite a los actos personales. Los motivos psicológicos y las ocasiones corpóreas pueden entrelazarse porque no se da ni un solo movimiento en un cuerpo vivo que sea un azar absoluto respecto de las intenciones psíquicas, ni un solo acto psíquico que no haya encontrando cuando menos su germen o su bosquejo general en las disposiciones fisiológicas... mediante una vuelta insensible, un proceso orgánico desemboca en un comportamiento humano, un acto instintivo vira y se vuelve sentimiento, o, inversamente, un acto humano entra en sueño y se prosigue distraídamente en reflejo (Merleau-Ponty, 1984: 106).

La condición humana de nuestra corporalidad hace que simultáneamente percibamos físicamente al mundo y lo vayamos significando a través de universos simbólicos cimentados en los procesos de incorporación cultural y social que van instituyendo el aspecto biológico del cuerpo (Le Bretón, 2007). Más que un organismo vivo, el cuerpo es una construcción social, cuyos procesos naturales, propios de su dimensión física y tangible, se van modificando a partir de los procesos de socialización de los primeros años de vida de un niño, de tal forma que las funciones corporales 
pasan a ser rápidamente transformadas por lo social (Soler, 2003:62). El cuerpo, entonces, va interpretando el mundo a través de las orientaciones culturales interiorizadas que nos han sido transmitidas por los procesos de socialización, en los cuales la sensación está inmersa en la percepción del mundo, y por lo tanto nuestra facultad de conocimiento del mundo está determinada por el sentido que le atribuyamos a éste, de tal manera que "lo que los hombres perciben no es lo real, sino ya un mundo de significados" (Le Bretón, 2007: 22).

\section{Implicaciones éticas del investigador en los procesos de investigación social}

Teniendo en cuenta las distintas y complementarias tendencias que se reconocen alrededor del cuerpo, se hace innegable aceptar que investigar el cuerpo suscita dificultades metodológicas permanentes que requieren de un esfuerzo continuo por integrar ambas dimensiones -biológica y socialdel cuerpo, no de manera paralela, sino, como se dijo anteriormente, como procesos que se estructuran mutuamente.

Aunque resulta interesante como tema a investigar, el cuerpo es complejo de comprender metodológicamente, debido a que en la vivencia cotidiana se va diluyendo en sensaciones imperceptibles que se pierden de la conciencia de nuestra corporalidad, por tal razón, quizá sea necesario incorporar una postura epistemológica más comprensiva para lograr percibir y entender sensitivamente el cuerpo, modificando la manera de pensar, de concebir y de vincularnos al mundo corporalmente (Le Bretón, 1994). Durante la mencionada investigación, se pudo vivenciar que existen límites difusos entre investigación e intervención social. Se evidenció que el distanciamiento aparente que se asume entre la investigación y la intervención se basa en las mismas dicotomías corporales que se hacen entre el pensamiento y la acción, es decir, entre la concepción del mundo y la capacidad de ejercer nuestra acción sobre él (Pakman, 1999).

En la visión tradicional, se operaba como si el investigador no fuera participante de aquello que pretende entender, describir y "descubrir" en su racionalidad oculta, y cree que recoge información y obtiene un 
conocimiento a posteriori. Sin embargo, la crisis epistemológica buscó evidenciar que los seres humanos nos relacionamos con el entorno a través de procesos de coparticipación entre observadores y observaciones, de tal forma que la investigación no es sólo una recolección de información acerca de algo, desde una posición distante, de observador, que precede a su intervención, ni la intervención es sólo un acto independiente de la información obtenida con anterioridad: ambos procesos se alimentan mutua y circularmente, y se vuelven dos modos posibles de descubrir una interacción como totalidad.

En una postura más constructivista, el investigador social no hace una reproducción de la realidad en el vacío, porque nunca está solo, sino que realiza una co-construcción del conocimiento en compañía de otros: autores, sujetos protagonistas y contexto, cuyo producto depende no sólo de las "perspectivas" y limitaciones del investigador (Santibañez, 1999 en Haefner, s.f.: 2), sino de los procesos de determinación y estructuración de quienes los detentan: "investigadores e investigados". Por lo tanto, se puede considerar que investigar "es un acto de intervención" porque la información se genera en la interacción resultante entre los participantes y tiene un efecto impredecible en el tiempo, creándose así un proceso social auto-referencial cuyo dominio participativo descubre el efecto de nuestra forma de concebir y de preguntar acerca del objeto de conocimiento sobre las personas y sus realidades (Pakman, 1999: 361).

De esta manera, los procesos de investigación social exigen la responsabilidad ética por parte del investigador de considerar los posibles efectos que se pueden movilizar en el otro "investigado" a partir de su indagación e interposición en su vida, y de comprender, al mismo tiempo, los vínculos que tiene su propia posición epistemológica frente a las determinaciones sociales donde convive (Jelin y Kaufman, 2006 en Aranguren, 2010).

Por lo tanto, la investigación y la intervención social, como procesos sociales, tienen desafíos similares, "que implican tanto el trabajo de elucidación y cambio como la producción de conocimientos": Quien interviene requiere del conocimiento de la realidad para adentrarse en proyectos de cambio, pero, al mismo tiempo, las problemáticas sociales 
en las que acciona y su práctica misma de intervención pueden convertirse en objeto de conocimiento. Del mismo modo, el investigador cuya intencionalidad central es producir conocimiento puede generar ciertas consecuencias en el plano de la acción que trasciendan su tarea inicial y buscar que su producción "se convierta en el medio que habilita la elucidación y el cambio" de ciertas problemáticas sociales (Bedacarratx, 2002: 168-169).

Como se dijo anteriormente, uno de los desafíos al investigar el tema del cuerpo en los contextos de salud-enfermedad es el uso de cierta metodología que indaga discursivamente la experimentación corporal, limitada por nuestras propias características humanas y que diluye la vivencia cotidiana de nuestra corporalidad, porque justamente en el momento en que pensamos y hablamos sobre el cuerpo pasamos al plano abstracto y abandonamos su plano sensorial; al parecer, esto sucede en nuestra cultura occidental porque las manifestaciones corporales se aprenden a reprimir a partir de los procesos de socialización, como lo plantea Le Bretón (1990).

Por otra parte, concebir y estudiar el cuerpo como una presencia viva es considerar el tema de la incorporación de la experiencia perceptiva, es decir, la manera como un ser humano vive y participa en el mundo. Para Csordas, estamos viviendo "el final de una clase de cuerpo y el comienzo de otra clase de cuerpo", ${ }^{1}$ cuyo cambio fundamental está en la manera como nuestro cuerpo se organiza y experimenta el mundo (Csordas, 1994). Ni nuestros cuerpos personales, ni nuestros cuerpos sociales pueden ser vistos como algo natural que existe afuera del proceso de auto-creación denominado proceso humano.

Para Csordas (1994) y Sheper-Hughes (1995), la reflexión contemporánea acerca de la antropología del cuerpo debe fundamentarse en los procesos de incorporación (embodiment), una condición existencial para estar y habitar el mundo con el propio cuerpo (Pizza, 2005) que ayudaría a confrontar la posición dicotómica entre salud-enfermedad.

${ }^{1}$ Thomas J. Csordas, refiriéndose a los planteamientos que sugiere Emily Martin, afirma: "to the contemporary historical moment in which "we are undergoing fundamental changes in how our bodies are organized and experienced [...] we are seeing "the end of the kind of body and the beginning of another kind of body" (1992: 21, en Csordas, 1994: 1). 
Asimismo, la noción de agency, aportada por Gramsci, establece la relación entre el conocimiento y la praxis en los procesos de "transformación de la cultura y la sociedad", noción que, según Pizza, aún no ha sido redescubierta ampliamente en las ciencias sociales (2005: 18). La consideración que hace Gramsci acerca de la cultura como "un pensamiento en acción" y como un "proceso dialéctico de conocimiento, producción y transformación del yo" en el contexto histórico, ayudaría a converger aquellos límites entre la producción del conocimiento y la intervención que han permanecido distantes durante mucho tiempo.

Para Gramsci, la antropología debe poder estudiar al hombre como un producto histórico en una vinculación directa con la vida práctica de los hombres, en la cual pensamiento y vida van juntos: "este esfuerzo continuo por elaborar una teoría viviente [...] más que una incesante actividad de estudio [...] le acompañó toda su vida" (Pizza, 2005: 20). Para él, la capacidad de creación política deviene de una teoría viviente que permita al mismo tiempo "comprender y sentir" el cuerpo, y reflejar "... en su constitución la experiencia concreta de la vida real", pero además "desde la que brote una actividad práctica, una iniciativa de voluntad, un diálogo de transformación" (p. 20).

Entonces, la producción de teoría tiene una dimensión política y puede contribuir a desvelar algunos límites presentes entre la producción de conocimiento y la intervención. En la medida en que los conceptos teóricos que conforman las teorías son construcciones naturalizantes y productos históricos, "siempre mudables", las teorías reflejan aquellas relaciones de fuerza y de poder de una sociedad y de un momento histórico; por lo tanto, si se logra declarar sus implicaciones y contradicciones en la experiencia real, se podrá unir las teorías a la "praxis transformadora" (p. 22). Será necesario entonces reconocer el carácter político que tiene la investigación social frente a los procesos de determinación y estructuración de quienes la detentan: "investigadores e investigados", ya que la producción del conocimiento es fruto de la co-construcción de ambos y se constituye en un dispositivo de reproducción de lo social, al margen de esos procesos (Haefner, s.f.).

En este sentido, el trabajo de campo en la investigación social se puede considerar como un tipo de intervención cuando se realiza una permanente 
reflexión "no sólo del tipo de material recogido/construido, sino también sobre la experiencia vivida y las constantes tensiones surgidas entre lo esperado y lo encontrado en "el campo" (Bedacarratx, 2002: 156). Esta reflexión ética, a partir de enfoques fenomenológicos y constructivistas sociales, conlleva a pensar en el tema de la inclusión del investigador, es decir, en las sensaciones y emociones surgidas en él durante el proceso, su implicación y el efecto de su indagación sobre los otros; ésta se convierte en la fuente de la reflexión crítica y ética que debe emprender. El investigador está "indisociablemente unido" no sólo con el objeto de estudio, sino también con el terreno en donde se lo aborda, por lo tanto, su tarea ética será reflexionar sobre ello, comprender su propia posición, los vínculos con las determinaciones que se tengan, explicitar su propia postura y su mirada frente al otro (p. 157).

Además, tanto el campo de la investigación como el de la intervención están imbuidos por desafíos "que implican tanto el trabajo de elucidación y cambio como la producción de conocimientos", básicamente porque es imposible eliminar la ambigüedad existente entre producción de conocimientos y proyecto de cambio; ambos pueden trascender su intencionalidad inicial:

El consultor cuya intencionalidad es esencialmente praxeológica puede transformar su práctica de intervención y los fenómenos desencadenados en objeto para la producción de conocimientos [...] Del mismo modo, el investigador cuya intencionalidad central es la de producción científica de conocimiento puede obtener el "beneficio añadido" al acarrear ciertas consecuencias en el plano de la acción [...] puede "elevar" su aspiración de producir conocimiento, buscando que esta tarea de producción se convierta en el medio que habilita la elucidación y el cambio (p. 168-169).

La noción de implicación señala que toda "producción teórica no sólo está condicionada epistemológicamente, sino que también lo está institucional y socialmente" (Bedacarratx, 2002: 157). Según Ardoino (1980), tener en cuenta la subjetividad del investigador es entender que la producción de conocimiento siempre está sesgada; más que un obstáculo, la subjetividad, ayuda a comprender la relativización de "nuestras verdades, 
posibilitando la reflexión sobre nuestro propio proceso de entendimiento" (p. 157). Es decir, el lugar que ocupamos y nuestras dimensiones valorativas y afectivas suscitadas en el momento en que investigamos nos ayudan a ser conscientes de cómo condicionamos la producción de conocimiento.

Por otro lado, "al ingresar [la investigación] al campo empírico, campo histórico-social de los sujetos, al interrogarlos, interpelarlos, queda comprometida una promesa de "respuestas" que no se puede/debe eludir, estableciéndose un compromiso tácito con el otro que requiere reflexión y acuerdos sobre lo que se va a hacer con el material recogido, cómo se va a utilizar y para qué les va a servir (Garay, 2000: 20). En consonancia, este compromiso político y ético en la producción de conocimiento no descansa sólo en la intención de la aplicación de sus resultados a determinado contexto social, sino en la reflexión suscitada en el trasegar de la investigación.

El investigador puede experimentar una serie de sentimientos producto de la escucha de aquellos testimonios de dolor y sufrimiento incorporados en los procesos de salud-enfermedad que deben usarse para reflexionar sobre este compromiso ético y moral con el otro. Scheper-Hughes (1995), en su artículo sobre La primacía de la ética, discurre sobre el deber ético que implica para el investigador hacerse parte de lo que pretende "estudiar"; ella habla de una antropología womanly-hearted, en la cual importa no sólo lo que piensen los seres humanos, sino cómo se comportan el uno hacia el otro.

Como lo plantea la autora, constantemente las personas que participan en una investigación esperan algo de quien está observando y escuchando, $\mathrm{y}$ en ocasiones se explicita la intención de ayuda, una especie de acompañamiento frente a las situaciones que los invaden; precisamente, éste es el momento en que el investigador debe asumir su posición comprometida ética y políticamente con las personas. Para el caso de América Latina, dice la autora, el choque que esta idea causa es menor que en otros países, porque acá "el proyecto del antropólogo es al mismo tiempo etnográfico, epistemológico y político"2 (Scheper-Hughes, 1995: 415-416).

2 "The idea of an active, politically committed, morally engaged anthropology strikes many anthropologists as unsavory, tainted, even frightening. This is less so in parts of Latin America, India, and Europe (Italy and France, for example), where the anthropological project is at once ethnographic, epistemological, and political and where anthropologists do communicate broadly with "the polis" and "the public". 
Sin embargo, cuando el investigador aún no ha trasegado por los avatares invisibles que tienen la investigación y la intervención, este proceso puede resultar un tanto infructuoso. Las expectativas del investigador y de los participantes de la investigación pueden no coincidir. En un extremo podrían ubicarse aquellas personas que aceptan participar en una investigación porque tienen intereses de transformación y cambio frente a una situación en particular y asumen que el investigador aportará algo a sus vidas, algo que les permita hacer virar el sufrimiento. Estas personas pueden encontrarse con un investigador ubicado aún en su papel tradicional: "neutral, frío, racional y observador" de la condición humana (p. 410), que concibe como separados el conocimiento y la práctica social. Éste actuará de manera confusa, temerosa, frente a las expectativas de las personas con quienes trabajará en la investigación. En el otro extremo puede estar aquel otro investigador-interventor que logra entender que al escuchar al otro, observarlo, mirarlo, tocarlo y abrazarlo con compasión y empatía, realiza actos de solidaridad tan tremendamente significativos que logran convertirse en un trabajo de reconocimiento y de liberación humana (Scheper-Hughes, 1995). Parafraseando a la autora, si los profesionales de las ciencias sociales "niegan el poder para identificar una enfermedad o un mal y eligen ignorar en toda su extensión, juegan un papel de dominación sobre ellos, y colaboran con las relaciones de poder y de silencios que permiten que la destrucción continúe"3 (pp. 418-419). Hablar de la primacía de la ética, para Scheper-Hughes, es definir la ética como precultural, en la medida en que nuestra existencia humana como seres sociales presupone la presencia del otro.

El investigador es una especie de testigo responsable del testimonio que ha recibido del otro porque investigar es un acto moral que lo obliga a hacerse responsable de lo que escucha, de lo que ve, de lo que no ve, de lo que hace, de lo que no hace, de cómo lo hace, pero también de lo que escribe sobre los otros. A propósito, De Certeau (2000) considera que escribir es una "actividad concreta que consiste en construir, sobre un espacio propio,

3 "If anthropologists deny themselves the power (because it implies a privileged position) to identify an ill or a wrong and choose to ignore (because it is not pretty) the extent to which dominated people sometimes play the role of their own executioners, they collaborate with the relations of power and silence that allow the destruction to continue". 
la página, un texto que tiene poder [...] tiene como "sentido" remitir a la realidad $[\ldots]$ con vistas a cambiarla. Pretende una eficacia social" (p. 149).

El testimonio del otro en situaciones extremas, como la violencia o el sufrimiento, tiene un lugar central en la reflexión y en la práctica disciplinaria (Das, 2008) porque "el testimonio no es simplemente una herramienta metodológica para satisfacer la curiosidad intelectual; es, ante todo, una forma de dar cuenta de las experiencias de los protagonistas [...] sin perder de vista el sentido del evento" (Ortega, 2008: 40). Darle al investigador un testimonio de la vida propia y de experiencias dolorosas o situaciones extremas vividas es un acto de por sí liberador y puede tener un efecto reparador y terapéutico, de acuerdo con lo que plantea Lara (2002), al estudiar el concepto de juicio en Hannah Arendt:

El que dice lo que existe [...] siempre narra algo, y en esa narración los hechos particulares pierden su carácter contingente y adquieren cierto significado humanamente captable. Es bien cierto que "todas las penas se pueden sobrellevar si las pones en un cuento o relatas un cuento sobre ellas", como dijo Isak Dinessen [...] Podría haber añadido que incluso la alegría y la dicha se vuelven soportables y significativas para los hombres sólo cuando pueden hablar sobre ellas y narrarlas como un cuento. Hasta donde es también un narrador, quien dice la verdad factual origina esa "reconciliación con la realidad" que Hegel [...] comprendió como el fin último de todo pensamiento filosófico (Arendt, 1996: 275 en Lara, 2002: 28).

El ingrediente necesario para que el efecto de dar testimonio de la vida personal sea beneficioso y "político" es "tomar en cuenta" el sentido de lo que el hablante dice. Como lo argumenta Ortega, parafraseando a Das, es necesario entender el testimonio "desde la cotidianidad de los hablantes, anclados en procesos subjetivos y colectivos, estructurados por tradiciones simbólicas y encauzados por géneros discursivos. Una lectura atenta del testimonio debe abrirnos simultáneamente a la cotidianidad del acontecimiento y al testimonio en tanto acontecimiento" (Ortega, 2008: 40).

Das considera que el dar testimonio al otro se relaciona con tres funciones, que pueden considerarse con carácter terapéutico: la primera función está relacionada con la posibilidad de nombrar lo padecido, poner en palabras el dolor y el sufrimiento; la segunda se refiere al hacer, cuya 
connotación puede interpretarse como la posibilidad de iniciar un proceso de reelaboración de la situación, que ella denomina duelo; y la tercera se relaciona con la importancia que tiene establecer una relación con los otros, en este caso con el investigador, cuya relación debe permitir la legitimización y la contemplación del otro.

A esta narratividad, como forma discursiva, se le atribuyen cualidades terapéuticas porque es una modalidad fundamental para "aprehender y darle sentido a nuestro entorno [...] integra diversos elementos de la trama, potencia su capacidad significativa, produce coherencia social y conduce o sugiere una resolución de los conflictos elaborados [...] moralizamos la realidad" (Danto, 1985: xiii). Sin embargo, esta modalidad es válida sólo si se considera "la narrativa como acto social" que incita la ejecución de acciones con sentido para ayudarlos a la apropiación de su vida (Ortega, 2008: 44).

Pero además, esta narratividad, en el caso del estudio del cuerpo en contextos de salud-enfermedad, debe estar acompañada de un repertorio corporal que ayude a contextualizar mejor la palabra, suplementarla, mostrando el dolor en el cuerpo. Esa narratividad discursiva y corporal requiere de un investigador que escuche empáticamente y que se acerque a sentir y recibir el dolor del otro, porque aquella persona que investiga el cuerpo no sólo debe saber "con el intelecto, sino con las emociones" (p. 55).

\section{Conclusiones}

- El cuerpo no puede concebirse en oposición a las dimensiones físicabiológica y socio-cultural, pues adquiere un lugar simbólico en el lenguaje y en la comunicación, que evidencia la compleja relación entre la naturaleza y la cultura.

- Las diversas tendencias teóricas para comprender el cuerpo como categoría analítica han ido modificándose a través de ciertos cambios epistemológicos. Aunque aún permanece la visión dicotómica del cuerpo que consolida teorías que dividen la corporalidad del estudio de lo social, existen otros trabajos filosóficos y antropológicos de carácter fenomenológico y constructivista que permiten considerar la corporalidad como constitutiva de lo humano. Desde la 
Antigüedad, el cuerpo ha ido recibiendo diversas fuerzas y discursos que ejercer un peso sobre él y lo han ido configurando socialmente. Algunos estudios históricos se han ocupado de comprender los procesos de civilización de la sociedad occidental, en los cuales los seres humanos y su cuerpo se han visto sometidos a una serie de restricciones limitadas que atenúan los excesos corporales y afectan el tipo de relación que se establece entre los seres humanos. Sin embargo, existen otros estudios que se ocupan no sólo de comprender tales determinaciones sociales que se ejercen sobre el cuerpo, sino de aquellas fronteras extremas en las que los discursos sociales quedan restringidos y las personas escapan a tales determinaciones invisibles, a través de procesos de creación y autonomía cotidianas, necesarias para repensar el lugar del sujeto como actor de su propia historia.

- La investigación social tiene la responsabilidad ética de emprender reflexiones críticas que logren poner en evidencia tales determinaciones sociales presentes en la subjetividad del investigador y en las valoraciones que hacemos de la realidad social que investigamos. Pero además, nuestro compromiso político y ético como investigadores sociales se sustenta en la posibilidad de lograr comprensiones corporales más constitutivas y sociales de los sujetos, que integren una posición de escucha frente al otro, no sólo con la razón, sino con toda nuestra corporalidad, utilizando nuestra subjetividad para reflexionar y validar la experiencia, el dolor y el sufrimiento del otro, asumiendo así un tipo de acompañamiento más humano.

- Finalmente, los límites visibles e invisibles entre investigación e intervención social nos invitan a continuar comprometiéndonos con aquellos desafíos cotidianos de lograr ir desarrollando esas "teorías vivientes" de las que hablaba Gramsci, fortaleciendo en los procesos de intervención social la gran capacidad de agencia que tenemos los seres humanos. 


\section{Referencias bibliográficas}

Aranguren, Juan Pablo (2010). "El investigador ante lo indecible y lo inenarrable (una ética de la escucha)". Revista Redbioética/UNESCO, Año 1, 1(2), pp. 111-124 [Versión electrónica]. Disponible en: http://www.unesco.org.uy/ shs/fileadmin/shs/redbioetica/revista_2/Aranguren1.pdf. Consultado: 10 de noviembre de 2011.

Ardoino, Jacques (1987). "La intervención, ¿Imaginario del cambio o cambio de lo imaginario?", en: Bedacarratx, Valeria (2002), “Implicación e intervención en la investigación social”. En. Tramas 18-19 UAM-X México, pp. 153-170 [Versión electrónica]. Disponible en: http://148.206.107.10/biblioteca_digital/ estadistica.php?id_host $=6 \&$ tipo $=$ ARTICULO\&id $=1110 \&$ archivo $=6-87$ 1110 bnt.pdf\&titulo=Implicación e intervención en la investigación social. Consultado: 21 de marzo de 2011.

Arendt, Hannah (1996). Verdad y política, Hannah Arendt, entre el pasado y el futuro: Ocho ejercicios de reflexión política, Barcelona: Península; en Lara, Laura Adrian (2002). El concepto de juicio en Hannah Arendt. VIII Congreso Español de Ciencia Política y de la Administración Política para un mundo en cambio. Grupo de Trabajo 01. Identidad cultural, racionalidad dialógica, comunidad: ¿Nuevas formas de neutralización de la política? Texto de la Maestría en Intervención Social. Universidad del Valle. 26 de marzo de 2010.

Bedacarratx, Valeria (2002), "Implicación e intervención en la investigación social”. En. Tramas 18-19 UAM-X México, pp. 153-170 [Versión electrónica]. Disponible en: http://148.206.107.10/biblioteca_digital/estadistica. php?id_host $=6 \&$ tipo $=$ ARTICULO\&id $=1110 \&$ archivo $=6-87-1110$ bnt. pdf\&titulo=Implicación e intervención en la investigación social. Consultado: 21 de marzo de 2011.

Citro, Silvia (2009). Variaciones sobre la corporalidad. En: Cuerpos Significantes. Travesías de una etnografia dialéctica. Buenos Aires: Biblos. Culturalia, pp. 43-82.

Csordas, Thomas J. (1994). Embodiment and experience. The existential ground of culture and self. Cambridge studies in medical anthropology [Version electrónica]. Disponible en: http://books.google.com/books?id=24TAxcRJES QC\&printsec $=$ frontcover\&dq $=$ thomas + csordas\& $\mathrm{hl}=$ es\&ei $=$ MYe8Ts7FKoXm 0 QHNhvXrBA\&sa $=$ X\&oi=book_result\&ct $=$ result\&resnum $=1 \&$ sq $i=2 \& v e d=0$ CCwQ6AEwAA\#v=onepage \&q\& $\mathrm{f}=$ false. Consultado: 20 de octubre de 2011 .

Danto, Arthur C., Narration and knowledge (New York: Columbia University Press, 1985), pp. xiii; 143-181. En: Ortega, Francisco (ed.), 2008. Veena Das. Sujetos de dolor, agentes de dignidad. Bogotá, Universidad Nacional de Colombia. 
Das, Veena (1996). "Lenguaje y cuerpo: transacciones en la construcción del dolor". En: Ortega A., Francisco. Colección Lecturas CES. Universidad Nacional de Colombia. Facultad de Ciencias Humanas. Centro de Estudios Sociales, pp. 343-373.

(2008). "Sujetos de dolor, agentes de dignidad". Francisco Ortega (Editor). Universidad Nacional de Colombia. Bogotá.

De Certeau, Michel (2000). La invención de lo cotidiano 1. Artes del hacer. México: Universidad Iberoamericana. Instituto Tecnológico y de Estudios Superiores de Occidente.

Elias, Norbert (1966). La sociedad cortesana. México: Fondo de Cultura Económica.

(1993). Entrevista en civilización y subjetividad. Zona Erógena, N. ${ }^{\circ}$

13 [Versión electrónica]. Disponible en: http://hyperelias.jku.at/Abstracts/ Fulltext-spa-1993-T-spa-1.pdf Consultado: 24 de enero de 2012.

Foucault, Michel (1966). El nacimiento de la clínica. Una arqueología de la mirada médica. México: Siglo Veintiuno.

(2006). Historia de la sexualidad. 3. El cuidado de sí. IV. El Cuerpo. Madrid: Siglo XXI, pp. 113-167.

Garay, Lucía (2000). "Investigar en educación hoy es intervenir". En: Revista Cuadernos de Educación, Córdoba, Año 1, N. ${ }^{\circ}$ 1, pp. 19 - 23. Disponible en: $\quad$ http://revistas.unc.edu.ar/index.php/Cuadernos/article/view/621/585. Consultado: 5 de mayo de 2011.

García, Fernando J. (1994), El "cuerpo" como base del sentido de la acción. Revista Española de Investigaciones Sociológicas REIS 68, octubre-diciembre, pp. 41-83.

Haefner, Carlos (s.f.). "La investigación e intervención social como sistemas de observación de segundo orden" [Versión electrónica]. Disponible en: http:// tsocial.ulagos.cl/redescuelas/doc/elzs-01-007.doc Consultado: 8 de marzo de 2011.

Jelin, Elizabeth y Kaufman, Susana (2006). "Diálogos intergeneracionales en un grupo de investigación sobre memorias: algunas reflexiones". En: Jelin, Elizabeth y Kaufman, Susana (comp.). Subjetividad y figuras de la memoria, Buenos Aires y Nueva York, Siglo XXI Editora Iberoamericana y Social Science Research Council, pp. 183-197. En: Aranguren, Juan Pablo (2010). "El investigador ante lo indecible y lo inenarrable (una ética de la escucha)". Revista Redbioética/UNESCO, Año 1, 1(2), pp. 111-124 [Versión electrónica]. Disponible en: http://www.unesco.org.uy/shs/fileadmin/shs/redbioetica/ revista_2/Aranguren1.pdf. Consultado: 10 de noviembre de 2011.

Kleinman, Arthur (1988). The Illness Narratives. Suffering, Healing, and the Human Condition. United States of America: Basic Books. 
Lara, Laura Adrian (2002). El concepto de juicio en Hannah Arendt. VIII Congreso Español de Ciencia Política y de la Administración Política para un mundo en cambio. Grupo de Trabajo 01. Identidad cultural, racionalidad dialógica, comunidad: ¿Nuevas formas de neutralización de la política?, texto de la Maestría en Intervención Social. Universidad del Valle. 26 de marzo de 2010.

Le Bretón, David (1990). Antropología del cuerpo y Modernidad. Buenos Aires: Nueva Visión.

(1994). "Lo imaginario del cuerpo en la tecnociencia". Revista Española de Investigaciones Sociológicas REIS 68, octubre-diciembre, pp. 197-210.

(2007). "Una antropología de los sentidos, de ver a saber". En: El sabor del mundo. Una antropología de los sentidos. Buenos Aires: Nueva visión, pp. 19-91.

Mauss, Marcel (1979). "El concepto de la técnica corporal, Principios clasificatorios de las técnicas corporales”. En: Sociología y Antropología. Madrid: Tecnos, pp. 337-356.

Merleau-Ponty, Michael (1984). Fenomenología de la percepción. Barcelona: Planeta-Agostini.

Ortega, Francisco (ed.) (2008). Veena Das. Sujetos de dolor, agentes de dignidad. Bogotá: Universidad Nacional de Colombia.

Pakman, Marcelo (1999). "Investigación e intervención en grupos familiares. Una perspectiva constructivista”. En: Juan Manuel Delgado y Juan Gutiérrez. Métodos y técnicas cualitativas de investigación en ciencias sociales Madrid: Síntesis, pp. 359-377.

Pedraza, Zandra (2003). Cuerpo e investigación en teoría social. Departamento de Antropología. Universidad de los Andes. Octubre de 2003 [Versión electrónica]. Disponible en http://antropologia.uniandes.edu.co/zpedraza/zp1. pdf. Consultado: 20 de abril de 2010.

Pizza, Giovanni (2005). “Antonio Gramsci y la antropología médica contemporánea. Hegemonía, "capacidad de actuar" (agency) y transformaciones de la persona". En: Revista de Antropología Social, N. ${ }^{\circ} 14$, pp. 15-32.

Reyes A. Ramfis y Eroza S. Enrique (2008). "El cuerpo y las ciencias sociales". En: Revista pueblos y fronteras digital. La noción de persona en México y Centroamérica, N. ${ }^{\circ}$ 4, dic. 2007-may. 2008, pp. 1-56 [Versión electrónica]. Disponible en: http://www.pueblosyfronteras.unam.mx/a07n4/pdfs/n4_art02. pdf. Consultado: 20 de abril de 2010.

Salinas, Lola (1994). "La construcción social del cuerpo". Revista Española de Investigaciones Sociológicas REIS 68, octubre-diciembre, pp. 85-96.

Santibañez, Dimas (1997). "Investigación social y autorreferencia. Sistemas sociales y sistemas psíquicos como sistemas autorreferenciales" [Versión electrónica]. Disponible en: http://redalyc.uaemex.mx/pdf/101/10100207. 
pdf. En: Haefner, Carlos (s.f.). "La investigación e intervención social como sistemas de observación de segundo orden”. [Versión electrónica]. Disponible en: http://tsocial.ulagos.cl/redescuelas/doc/elzs-01-007.doc. Consultado: mayo de 2011.

Scheper-Hughes, Nancy (1995). The Primacy of the Ethical. Propositions for a Militant Antrhropology. University of California - Berkeley. Permalink [Versión electrónica] Disponible en: http://escholarship.org/uc/item/2xq430hc.

Sierra H., Carlos Hugo (2009). "Regímenes de producción de cuerpos en el espacio médico". En: Mendiola G., Ignacio (ed.). Rastros y rostros de la biopolítica. Barcelona: Anthropos, pp. 223-246.

Soler Colette. (2003). LeTrazas 3. V. Cuerpos Contemporáneos. "El cuerpo, acontecimiento de discurso" Bogotá, pp. 62-68.

Suárez M., Roberto (2001) (comp.). Salud-enfermedad: una categoría a repensar desde la antropología. En: Reflexiones en salud: una aproximación desde la antropología. Bogotá: Ceso. Ediciones Uniandes. Departamento de Antropología. Universidad de los Andes, pp. 11-21. 